\title{
Positive Charge Transport in an Alternating Oligo-p-phenylenevinylene-derivative/aliphatic-segment Block Copolymer
}

\author{
A.R.V. Benvenho, I.A. Hümmelgen* \\ Departamento de Física, Universidade Federal do Paraná \\ C.P. 19044, 81531-990 Curitiba - PR, Brazil
}

Received: January 10, 2001; Revised: March 26, 2001

\begin{abstract}
An effective positive charge carrier mobility of $4.4 \times 10^{-9} \mathrm{~cm}^{2} / \mathrm{V} . \mathrm{s}$ was directly determined using analytical expressions for space-charge-limited current in poly(1,10-decanedioxy-2-methoxy1,4-phenylene-1,2-ethenylene-3,6-dimethoxy-1,4-phenylene-1,2-ethenylene-3-methoxy-1,4-phe nylene), OPPVDBC, which is a soluble electroluminescent alternating block copolymer. Current vs. voltage measurements have been made using single carrier injection and transport in metal/OPPVDBC/metal structures. The experimental results demonstrate that the positive charge transport in these devices is space-charge-limited and temperature dependent. Additionally, there is evidence that the evaporation of the aluminum electrode induces an increase in the charge density near the Al/OPPVDBC interface.
\end{abstract}

Keywords: block copolymer, electroluminescent polymer, charge carrier mobility

\section{Introduction}

Conjugated oligomers have well defined optical emission and absorption characteristics, which make them natural candidates for organic optoelectronic applications ${ }^{1}$. In spite of their interesting optical properties, these organic semiconductors commonly present difficulties when used in the form of thin films, due to their low molecular weight. High molecular weight materials such as polymers may present good mechanical properties, from which stable homogeneous, large area thin films can be obtained, even through technologically quite simple processes. A strategy that permits to combine the advantages of both classes of materials is to build an alternating block copolymer, constituted by conjugated segments (chromophores) and aliphatic segments (spacers). The chromophores determine the optical characteristics of the polymer, whereas the spacers may improve mechanical properties, e.g. flexibility, and enhance polymer solubility in common organic solvents. In this case, the spectral absorption/emission characteristics are controlled in a way similar to the variation of oligomer length ${ }^{2,3}$, simply controlling the length of the conjugated segment. Additional energy shifts (elec- troaffinity, ionization energy and energy gap) can be achieved through incorporation of substituents in the chromophores $^{4-6}$.

In the case of organic semiconductors, charge transport properties must also be considered in order to select promising candidates for device applications. Charge carrier mobility is a fundamental parameter that often controls the performance of pristine semiconductor polymer devices, its quantum efficiency, response time, current and light emission threshold ${ }^{7}$. Charge carrier mobilities have again an important role in achieving balanced injection of positive and negative charge carriers and in the spatial location of the emissive region in organic light emitting diodes ${ }^{8,9}$.

For metal/polymer/metal structures, depending on a careful choice of electrode materials, injection limited (interface controlled) charge transport can be avoided and the transport is in fact space-charge-limited ${ }^{8,10,11}$. When the current-voltage characteristics, $I(V)$, are space-charge-limited, the charge carrier mobility can be readily determined by space-charge-limited current measurements.

In this contribution we investigate the charge transport characteristics of a electroluminescent block copolymer (see Fig. 1) using the same procedure adopted to the char-

* e-mail: iah@fisica.ufpr.br 
acterization of several related copolymers investigated recently $^{12-16}$, allowing for a direct comparison of results. In the sequence, this copolymer will be designated OPPVDBC. Since the theoretical background used in this work is the same as reported in Refs. 12-16, a detailed description of space-charge-limited transport will be omitted here.

At low applied voltages the charge injected through the injecting electrode does not substantially disturb the equilibrium charge carrier concentration $n_{0}$ in the polymer, so that the transport is ohmic and the current density is described by ${ }^{17}$

$$
j=q \mu n_{0} \frac{V}{d}
$$

where $q$ is the elementary charge, $\mu$ is the charge carrier mobility and $d$ is the polymer film thickness.

At higher voltages and increased injection levels charge accumulates near the injecting electrode redistributing the electric field intensity, controlling charge transport through the polymer bulk and determining the I(V) dependence. In a trap-free conjugated polymer the space-charge-limited $j$ is expressed by ${ }^{17}$

$$
j=\frac{9}{8} \varepsilon \mu \frac{V^{2}}{d^{3}}
$$

where $\varepsilon$ is the permittivity of the polymer This charge transport case is characterized by a weak $\mu \mathrm{T}$ dependence (T: temperature). On the other hand, if traps present in the polymer layer are located at a single discrete energy level, this expression is modified to ${ }^{15}$

$$
j=\frac{9}{8} \varepsilon \mu \frac{N_{v}}{N_{t}} \exp \left(-\frac{E_{t}-E_{v}}{\kappa T}\right),
$$

so that the effective mobility can be written as

$$
\mu_{e f f} \equiv \mu \frac{N_{v}}{N_{t}} \exp \left(-\frac{E_{t}-E_{v}}{\kappa T}\right),
$$

yielding a strong temperature dependence for $\mu_{\text {eff }}$ and consequently, for I. Considering the specific case of positive charge carrier transport, $N_{v}$ is the density of HOMO (highest occupied molecular orbital) level states, $N_{t}$ is the density of charge trapping centers, $\mathrm{E}_{\mathrm{t}}$ is the trap center energy, $E_{v}$ is the energy of the mobility edge for

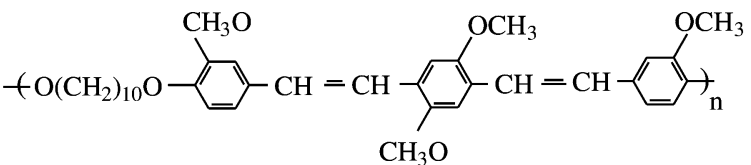

Figure 1. Structure of the alternating block copolymer used in this work, in poly(1,10-decanedioxy-2-methoxy-1,4-phenylene-1,2-ethenylene-3,6dimethoxy-1,4-phenylene-1,2-ethenylene-3-methoxy-1,4-phenylene), OPPVDBC. positive charge carriers and $k$ is the Boltzmann constant. Furthermore, in Eq. (1), $\mu$ must also be substituted by $\mu_{\text {eff }}$ to accomplish the single-discrete-trap case.

At the transition voltage $V_{\Omega}$ from ohmic to spacecharge limited case, the I(V) dependence follows simultaneously Eqs. (1) and (2) or their equivalents for the single-discrete-trap case (with $\mu_{\text {eff }}$ instead $\mu$ ). For this reason $\mathrm{V}_{\Omega}$ can be used to estimate $n_{0}{ }^{17}$ :

$$
n_{0}=\frac{9}{8} \frac{\varepsilon V_{\Omega}}{q d^{2}}
$$

In all the cases described above, $\mu$ (and also $\mu_{\text {eff }}$ ) is considered to be independent of the electric field strength though in some cases field dependent mobilities were also reported for several organic compounds ${ }^{18-21}$. But even in the case of field dependent mobility, this is typically observed only at high electric fields. For this reason, the mobility value determined in this work is the field-independent mobility.

\section{Experimental}

The metal/polymer/metal devices were prepared on glass substrates. The bottom metal electrode (Ni) was evaporated onto the glass and the polymer was spin coated from a $\mathrm{CHCl}_{3}$ solution $(5 \mathrm{mg} / \mathrm{mL})$ onto this electrode. The roughness $R a^{22}$ of these OPPVDBC films was approximately $10 \%$ of the sample thickness for the complete sample thickness range used $(25-150 \mathrm{~nm})$. The top metal electrode (Al) was then evaporated onto the polymer layer. These metals have work functions $\phi$ of $\sim 5.1 \mathrm{eV}$ and $\sim 4.3 \mathrm{eV}$, respectively. The polymer layer thickness in all devices was measured using a surface profiler. The currentvoltage measurements were made by increasing the voltage from zero up to the maximum value at a rate of $0.1 \mathrm{~V} . \mathrm{s}^{-1}$ and then decreasing to zero at the same rate. Details concerning sample geometry were as reported earlier ${ }^{23}$. The

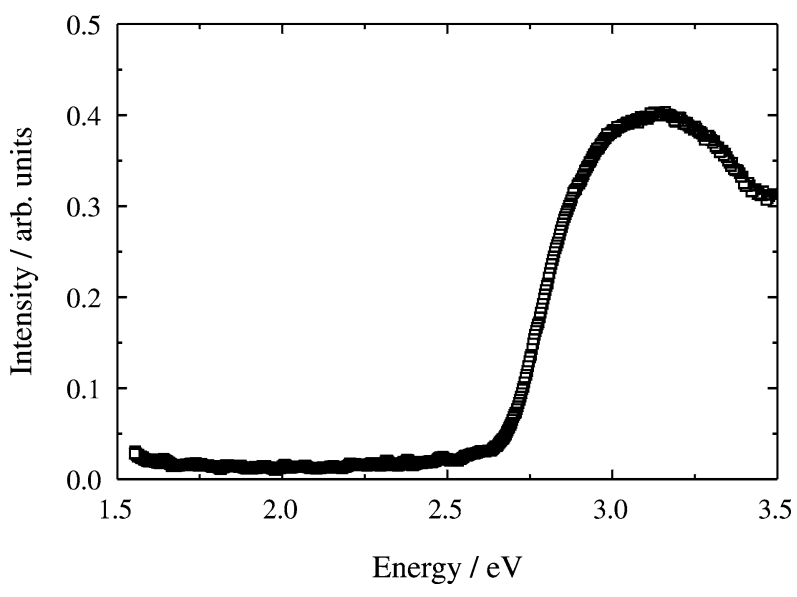

Figure 2. Absorption spectrum of OPPVDBC. 
permittivity of OPPVDBC was assumed to be $3 \varepsilon_{0}\left(\varepsilon_{0}\right.$ : permittivity of vacuum).

\section{Results and Discussion}

In Fig. 2 we present the UV-visible absorbance spectrum of the copolymer shown in Fig. 1. From the absorbance onset the energy gap of OPPVDBC can be estimated as being $\sim 2.6 \mathrm{eV}$.

The $j(\mathrm{~V})$ characteristics of a Ni/OPPVDBC/Al device show two defined regions, segments (a) and (b) (see Fig. 3). The first one (a) corresponds to ohmic behavior $(j \propto V)$, whereas in segment (b) $\mathrm{j} \propto \mathrm{V}^{2}$, suggesting space-chargelimited current. Fig. 4 shows the current dependence on the OPPVDBC layer thickness, at a constant voltage $\mathrm{V}=0.95$ V. A linear fit to these data gives $\partial \log I / \partial \log d=-3,13$, quite near the theoretically expected value, -3 . Since this value is determined using different samples (of different thickness) and that sample thickness may locally vary due to polymer film roughness, small deviations from the theoretical value, -3 , are commonly observed in experimental works ${ }^{12,15,16}$. The $\mathrm{I}(\mathrm{V}, \mathrm{d})$ dependence observed in $\mathrm{Ni} / \mathrm{OPPVDBC} / \mathrm{Al}$ clearly demonstrates that the charge carrier transport in these devices is space-charge-limited. Based on the electrode work function values and the electron affinity expected for these copolymers ${ }^{12}$, we conclude that $\mathrm{Ni} / \mathrm{OPPVDBC} / \mathrm{Al}$ devices operate in a hole-only mode (only positive charge carriers are injected and transported). Using Eq. (2) we determined the value of $\mu_{\text {eff }}$ taking the data corresponding to region (b). We obtained $\mu_{\text {eff }}=(4.4 \pm 0.5) \times 10^{-9} \mathrm{~cm}^{2} / \mathrm{V}$.s.

The temperature dependence of $\mu_{\text {eff }}$ is presented in Fig. 5. It corresponds to segment (b) of the $\mathrm{j}(\mathrm{V})$ curve of $\mathrm{Ni} / \mathrm{OPPVDBC} / \mathrm{Al}$ devices. The linear fit to the data of Fig. 4 results in $\partial \ln \mu / \partial T^{-1}=-194 \mathrm{~K}^{-1}$, which corresponds to $\mathrm{E}_{\mathrm{t}}-\mathrm{E}_{\mathrm{v}}=0.016 \mathrm{eV}$ [see Eq. (4)].

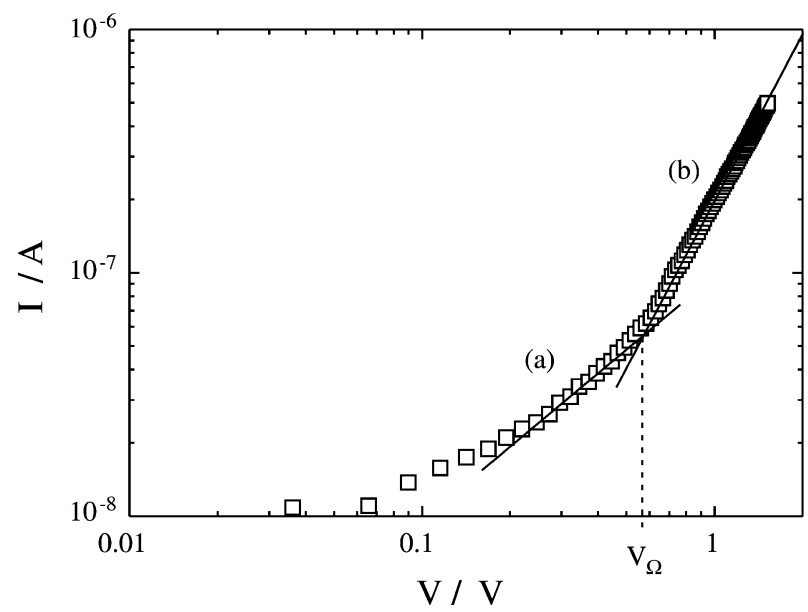

Figure 3. I(V) curve of an Ni/OPPVDBC/Al device. Device area: $1 \mathrm{~mm}^{2}$; OPPVDBC layer thickness: $92 \mathrm{~nm}$; Ni positively biased.

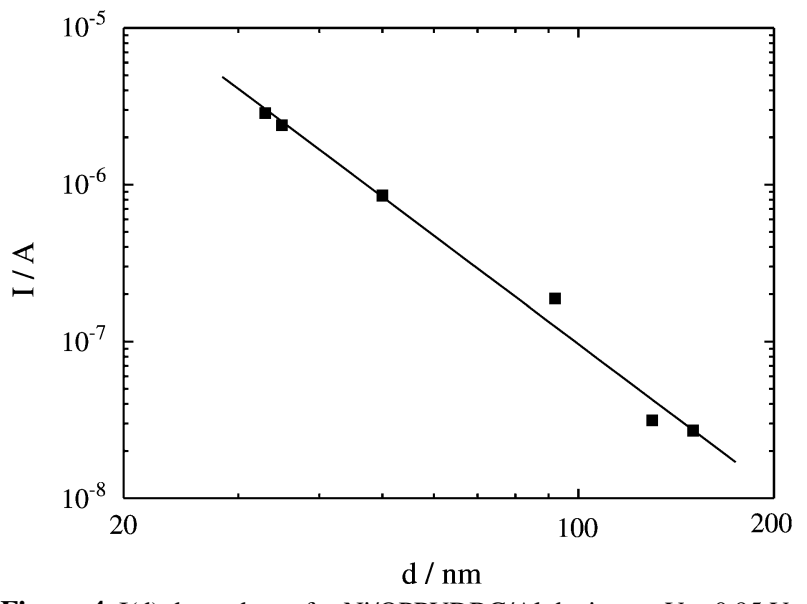

Figure 4. I(d) dependence for Ni/OPPVDBC/Al devices at V $=0.95 \mathrm{~V}$.

We could not find a proof to attribute this temperature dependent effective mobility to charge traps. Our samples do not withstand voltages higher than those applied. If higher voltages the devices suffered degradation. For this reason we could neither find a trap-filling region ${ }^{12}$ nor a hysteresis in the increasing-voltage decreasing-voltage $\mathrm{I}(\mathrm{V})$ curve, which would allow us to distinguish between another thermally activated conduction mechanism and a trap population controlled mobility, in favor of the latter. An exponential $\mathrm{I}(\mathrm{T})$ dependence can also be originated through disorder induced self-trapping of charge carriers in the polymer and it is expressed as a field dependent mobility ${ }^{20}$, normally at higher electric fields, which could not be reached. However, it was observed that energetic disorder is much more pronounced in similar block copolymers with shorter aliphatic groups ${ }^{15}$. In such copolymers disorder parameters are also strongly temperature dependent ${ }^{15}$. OPPVDBC takes advantage of a long aliphatic segment, just reducing energetic disorder.

Our results can be compared with previous data on a similar copolymer, which contains the same spacer but with an $\mathrm{OCH}_{3}$ bi-substituted chromophore (the bi-substituted

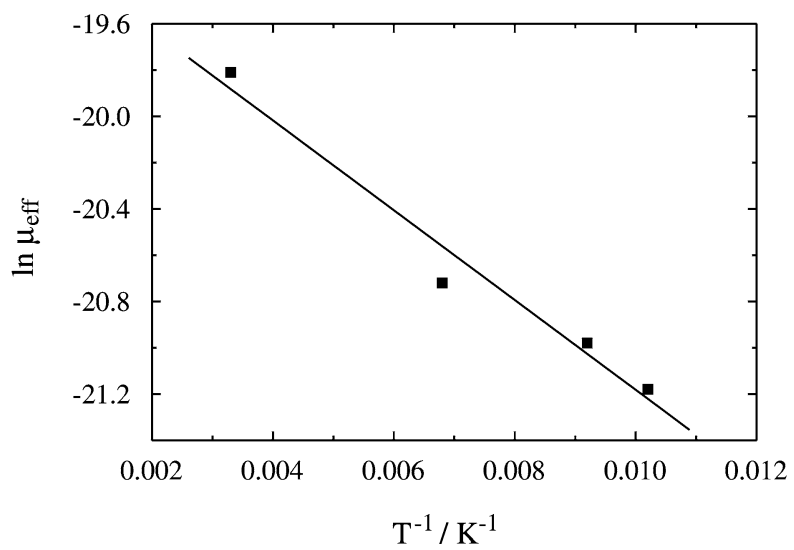

Figure 5. Dependence of $\mu_{\text {eff }}$ on temperature. Ni/OPPVDBC/Al device; Device area: $1 \mathrm{~mm}^{2}$; OPPVDBC layer thickness: $130 \mathrm{~nm}$. 


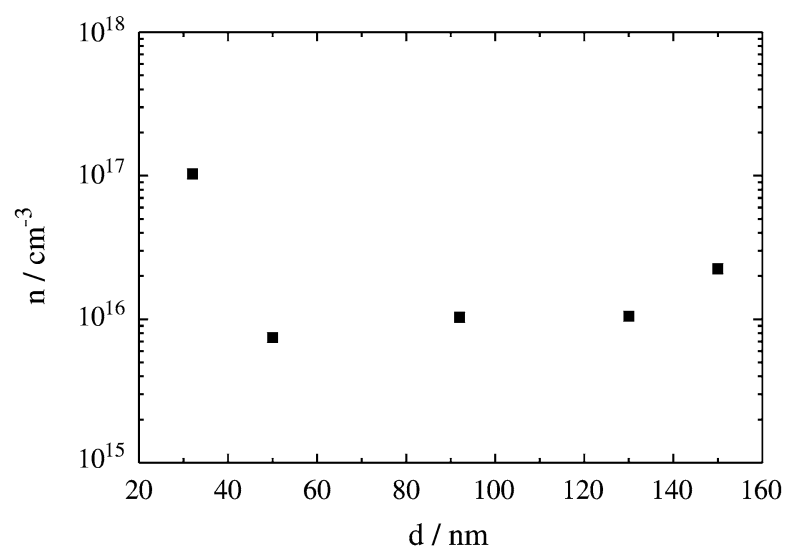

Figure 6. Charge carrier density in Ni/OPPVDBC/Al devices as a function of device thickness.

chromophore block copolymer presents $\mathrm{OCH}_{3}$ groups only at the central chromophore ring $)^{16}$. The bi-substituted chromophore block copolymer has a positive charge carrier mobility of $1.310^{-10} \mathrm{~cm}^{2} / \mathrm{V}$.s before trap filling and $1.4 \times 10^{-8} \mathrm{~cm}^{2} / \mathrm{V} . \mathrm{s}$ after trap filling. The mobility of OPPVDBC lies in this range, so that no definite conclusion about the consequences of introduction of two additional $\mathrm{OCH}_{3}$ groups in the chromophore on copolymer charge carrier mobility can be inferred.

In Fig. 6 we present $n_{0}$ as a function of sample thickness, determined using Eq. (5). It can be seen that except for the thinner sample, the value of $n_{0}$ remains nearly independent of $d$, around $10^{15} \mathrm{~cm}^{-3}$ at room temperature. The fact that thin samples present a higher $n_{0}$ value suggests that the interface neighborhood is affected during the top metal contact evaporation process inducing the appearance of additional energy levels that may increase the charge carrier concentration in the metal electrode vicinity ${ }^{24}$.

This observation is important since electrode evaporation is a necessary step in device preparation. With additional polymer-layer-depth-dependent disorder originated from the solvent evaporation, the polymer film charge transport properties can, depending on polymer, solvent and electrode preparation process, be position dependent and, as a consequence, also thickness dependent ${ }^{25}$.

\section{Conclusion}

Current $v s$. voltage measurements have been made using single carrier (positive) injection into metal/OPPVDBC/metal structures. OPPVDBC is a soluble alternating block copolymer. Our experimental results demonstrate that the positive charge transport in $\mathrm{Ni} / \mathrm{OPPVDBC} / \mathrm{Al}$ is space-charge-limited and temperature dependent. The effective positive charge carrier mobility of $4.410^{-9} \mathrm{~cm}^{2} / \mathrm{V}$.s was directly determined using spacecharge-limited current analytical expressions. We also found evidences that the evaporation of the aluminum electrode induces an increase in the charge density $n_{0}$ near the Al/OPPVDBC interface.

\section{Acknowledgments}

This work was supported by CNPq/PADCT under project 62.0081/97-0 CEMAT. ARVB thanks CAPES for a scholarship and IAH thanks CNPq for a research fellowship. The authors would like to thank Dr. Dongge Ma and Dr. Zhyiong Hong for polymer samples.

\section{References}

1. Hutten, P.F. van; Hadziioannou, G. Semiconducting Polymers, Hadziioannou, G.; Hutten, P.F. van, eds., Wiley-VCH, p. 561, 2000.

2. Geerts, Y.; Klärner, G.; Müllen, K. Electronic Materials: The Oligomer Approach, Müllen, K.; Wegner, G., eds., Wiley-VCH, p. $1,1998$.

3. Pope, M.; Swenberg, C. E.. Electronic Processes in Organic Crystals and Polymers, Oxford University Press, p. 5, 1999.

4. Brédas, J.L.; Cornill, K.; Meyers, F.; Beljonne, D. Handbook of Conducting Polymers, Skotheim, T. A.; Elsenbaumer, R. L.; Reynolds, J.R.; Dekker, M., eds, p. 1, 1998.

5. Yang, Z.; Sokolik, I.; Karasz, F.E.. Macromolecules, v. 26, p. 1188, 1996.

6. Gurge, R.M.; Hickl, M.; Krause, G.; Lathi, P.M.; Hu, B.; Yang, Z.; Karasz, F.E. Polym. Adv. Technol., v. 9, p. 504, 1998.

7. Redecker, M.; Bradley, D.D.C.; Karun, M.I.; Wu, W.W.; Woo, E.P. Adv. Mater., v. 11, p. 241, 1990.

8. Koehler, M.; Luz, M.G.E. da; Hümmelgen, I.A. J. Phys. D: Appl. Phys., v. 33, p. 2096, 2000.

9. Grüner, J.; Remmers, M.; Neher, D. Adv. Mater., v. 9, p. 964, 1997.

10. Ma, D.; Hümmelgen, I.A.; Li, R.W.C.; Gruber, J. J. Phys. D: Appl. Phys., v. 33, p. 1376, 2000.

11. Koehler, M.; Hümmelgen, I.A. J. Appl. Phys., v. 87, p. 3074, 2000.

12. Dongge, Ma.; Hümmelgen, I.A.; Bin, H.; Karasz, F.E. J. Appl. Phys., v. 86, p. 3181, 1999.

13. Dongge, Ma.; Hümmelgen, I.A.; Hu, B.; Karasz, F.E. J. Phys. D: Appl. Phys., v. 32, p. 2568, 1999.

14. Dongge, Ma.; Hümmelgen, I.A.; Hu, B.; Karasz, F.E.; Jing, X.; Wang, L.; Wang, F. Sol. Stat. Comm., v. 112, p. 251, 1999.

15. Dongge, Ma.; Hümmelgen, I.A.; Jing, X.; Hong, Z.; Wang, L.; Zhao, X.; Wang, F; Karasz, F.E. J. Appl. Phys., v. 87, p. 312, 2000.

16. Dongge, Ma.; Hümmelgen, I.A.; Jing, X.; Wang, D.; Hong, Z.; Wang, L.; Zhao, X; Wang, F. Braz. J. Phys, v. 30, p.392, 2000.

17. Cao, K.C.; Hwang, W. Electrical Transport in Solids, Pergamon, 1981.

18. Bozano, L.; Carter, S.A.; Scott, J.C.; Malliaras, G.G.; Brock, P.J. Appl. Phys. Lett, v. 74, p. 1132, 1999.

19. Dunlap, D.H.; Parris, P.E.; Kenkre, V.E. Phys. Rev. Lett., v. 77, p. 542, 1996

20. Freire, J.A.; Luz, M.G.E. da; Ma, D; Hümmelgen, I.A. Appl. Phys. Lett., v. 77, p. 693, 2000.

21. Gruber, J.; Li, R.W.C.; Hümmelgen I.A. Handbook of Advanced Electronic and Photonic Materials and Devices, Nalwa, H. S., Academic Press, p. 163, 2001.

22. Whitehouse, D.T. Handbook of Surface Metrology, IOP, p. 14, 1994.

23. Roman, L.S.; Denicoló, I.; Nart, F.C.; Hümmelgen, I.A. J. Mater. Scienc. Lett., v. 15, p. 1307, 1996.

24. Simons, J.G. J. Phys. Chem. Solids, v. 32, p. 1987, 1971.

25. Koehler, M.; Luz, M.G.E. da; Hümmelgen, I. A. J. Phys. D: Appl. Phys., submitted. 